\title{
Identification of two proteins that interact with the Erp virulence factor from Mycobacterium tuberculosis by using the bacterial two-hybrid system
}

\author{
Laura I Klepp ${ }^{1}$, Marcelo Soria ${ }^{2}$, Federico C Blanco ${ }^{1}$, María V Bianco ${ }^{1}$, \\ María P Santangelo ${ }^{1}$, Angel A Cataldi ${ }^{1}$ and Fabiana Bigi*1
}

Address: ${ }^{1}$ Institute of Biotechnology, CICVyA-INTA Castelar, Nicolas Repetto and Los Reseros, 1686, Hurlingham, Argentina and ${ }^{2}$ Microbiología Agrícola Facultad de Agronomía. Universidad de Buenos Aires, Av. San Martin 4453, 1417 Buenos Aires, Argentina

Email: Laura I Klepp - lklepp@cnia.inta.gov.ar; Marcelo Soria - soria@agro.uba.ar; Federico C Blanco - fblanco@cnia.inta.gov.ar; María V Bianco - mbianco@cnia.inta.gov.ar; María P Santangelo - psantangelo@cnia.inta.gov.ar; Angel A Cataldi - acataldi@cnia.inta.gov.ar; Fabiana Bigi* - fbigi@cnia.inta.gov.ar

* Corresponding author

Published: 21 January 2009

BMC Molecular Biology 2009, 10:3 doi:10.1 186/1471-2199-10-3
Received: 20 June 2008

Accepted: 21 January 2009

This article is available from: http://www.biomedcentral.com/I47/-2/99//0/3

(c) 2009 Klepp et al; licensee BioMed Central Ltd.

This is an Open Access article distributed under the terms of the Creative Commons Attribution License (http://creativecommons.org/licenses/by/2.0), which permits unrestricted use, distribution, and reproduction in any medium, provided the original work is properly cited.

\begin{abstract}
Background: The exported repetitive protein (erp) gene encodes a secreted 36-kDa protein with a central domain containing several proline-glycine-leucine-threonine-serine (PGLTS) repeats. It has been demonstrated that erp is a virulence-associated factor since the disruption of this gene impairs the growth of Mycobacterium bovis and Mycobacterium tuberculosis in mice.

Results: In order to elucidate the function of Erp we searched for Erp-binding proteins from $M$. tuberculosis by using a bacterial two-hybrid system. Our results indicate that Erp interacts specifically with two putative membrane proteins, Rv/4I7 and Rv2617c. Further analysis revealed that the latter two interact with each other, indicating that Rv1417, Rv26I7c and Erp are connected through multiple interactions. While Rvl4I7 is disseminated in several Actinomycetales genera, orthologues of Rv2617c are exclusively present in members of the M. tuberculosis complex (MTC). The central and amino-terminal regions of Erp were determined to be involved in the interaction with RvI4I 7 and Rv2627c. Erp forms from Mycobacterium smegmatis and Mycobacterium leprae were not able to interact with Rv2617c in two-hybrid assays. Immunolocalization experiments showed that Rv1417 and Rv2617c are found on the cell membrane and Erp on the bacterial cell wall. Finally, comparative genomics and expression studies revealed a possible role of Rvl4I7 in riboflavin metabolism.
\end{abstract}

Conclusion: We identified interactive partners of Erp, an M. tuberculosis protein involved in virulence, which will be the focus of future investigation to decipher the function of the Erp family protein.

\section{Background}

M. tuberculosis Erp (Rv3810) and M. bovis P36 (Mb3840) are homologous $36 \mathrm{kDa}$ proteins that comprise 284 amino acids (aa) and possess a classical signal sequence. The central segment has 11 PGLTS repeats, four of which match exactly with the consensus and seven are degener- 
ate. The export signal sequence consists in four charged aa followed by 14 nonpolar ones and a probable cleavage site for the signal peptidase. Erp and P36 have been detected only in culture supernatants and cell wall preparations, but not in cell extracts [1-3].

De Mendoça et al. have demonstrated that orthologues of the erp gene are also present in saprophytic and environmental opportunistic pathogenic mycobacteria [4]. A striking feature of this family is that it has no orthologous sequences outside the Mycobacterium genus. Thus, it can be considered a Mycobacterium-specific family of secreted proteins.

Although the precise roles of Erp proteins have remained elusive, the number of reports showing that Erp is a crucial factor for survival and multiplication of bacteria both in vitro and in animal models is increasing. The initial evidence supporting a role of the Erp protein in mycobacterial pathogenesis came from a study by Berthet et al., who demonstrated that the disruption of erp/p36 in both $M$. tuberculosis and M. bovis BCG negatively affects the multiplication of these strains in infected cultured bone marrow-derived macrophages and mice [2]. In agreement with these results, disruption of $p 36$, impairs the growth of virulent M. bovis in vivo [5]. Finally, it has been reported that erp-deficient Mycobacterium marinum has an attenuated growth in cultured macrophage monolayers and during chronic granulomatous infection of leopard frogs, its natural host species. These results suggest that the function of Erp is similarly required for the virulence of $\mathrm{Myco-}$ bacterium species other than those belonging to the MTC [6]. It has also been shown that erp-deficient bacteria are attenuated primarily because of reduced intracellular growth and/or survival in macrophages from zebrafish embryos [6]. Thus, these findings reinforce the notion of Erp as a virulence factor of pathogenic mycobacteria. However, the exact function of this virulence factor during host infection is still unknown.

Because Erp has several central repeat regions, we hypothesized that these regions participate in the interaction with other proteins. In order to gain insights into the function of Erp, and based on the premise that the function of unknown proteins may be discovered through their interaction with a protein target with a known function, we searched for Erp-binding proteins from M. tuberculosis by using a bacterial two-hybrid system. We here report that Rv1417 and Rv2617c were able to interact with Erp and that these proteins relate to each other through multiple interactions. In addition, important aspects of the association of Erp with mycobacterial virulence are discussed.

\section{Results \\ I. The Erp protein interacts with Rv I4I 7 and Rv26 I7c in a bacterial two-hybrid system}

We used a two-hybrid system developed by Ladant and co-workers [7], in which genes of interest are fused to T18 and T25, two complementary fragments that are essential for adenylate cyclase activity. If the corresponding fusion proteins interact, cAMP is produced in an endogenous adenylate cyclase-deficient E. coli strain (BTH101), and this functional complementation can be easily monitored by plating bacteria in minimal medium supplemented with lactose. In this work, we searched for Erp-binding proteins by screening an $M$. tuberculosis DNA expression library with full-length Erp using this bacterial two-hybrid system. The size of the library was approximately $10^{5}$ clones. Out of $6 \times 10^{3}$ plated transformants, $10 \mathrm{cya}+$ clones which could grow in minimal medium supplemented with lactose were selected, indicating ten potential interactions. Enzymatic restriction analysis revealed that clones were unique (data not shown). In order to confirm the interactions and to exclude "false positives", plasmids were purified and used to retransform E. coli BTH101. In this second round, only three plasmid clones whose products were able to confer adenylate cyclase activity in E. coli BTH101 co-transformed with plasmid T25-Erp were selected. Sequence analysis of inserts revealed that two of these plasmids encoded Rv1417 and one Rv2617c. The plasmids encoding Rv1417 had a complete copy of the gene and they differed only in the length of the 5' region upstream of $R v 1417$, while in the plasmid encoding Rv2617c the first 60 bp of the gene were absent. Rv1417 and Rv2617c were annotated as membrane proteins, both of unknown function [8]. All sequenced fragments were in-frame with the ORF encoding T18. The fact that both plasmids encoding Rv1417 were independent clones confirms that non-redundant clones were present in the genomic library and reinforces the feasibility of ErpRv1417 protein interaction.

In order to examine the protein-protein interactions mentioned above, erp, $R v 1417$ and $R v 2617 c$ full-length genes were fused to both T18 and T25 gene sequences in pUT18c and pKT25 vectors, respectively. The efficiencies of functional complementation between hybrid proteins were determined by the number of colonies grown in M63 medium supplemented with lactose, and by $\beta$-galactosidase activity (see Additional file 1). The level of interaction between Erp and both Rv1417 and Rv2617c was significantly higher than that of the negative controls, independently of the adenylate cyclase fragments (T25 or T18) these proteins were fused to (Fig. 1). These interactions were confirmed by in vitro GST-Pull down assays (see Additional file 2). 


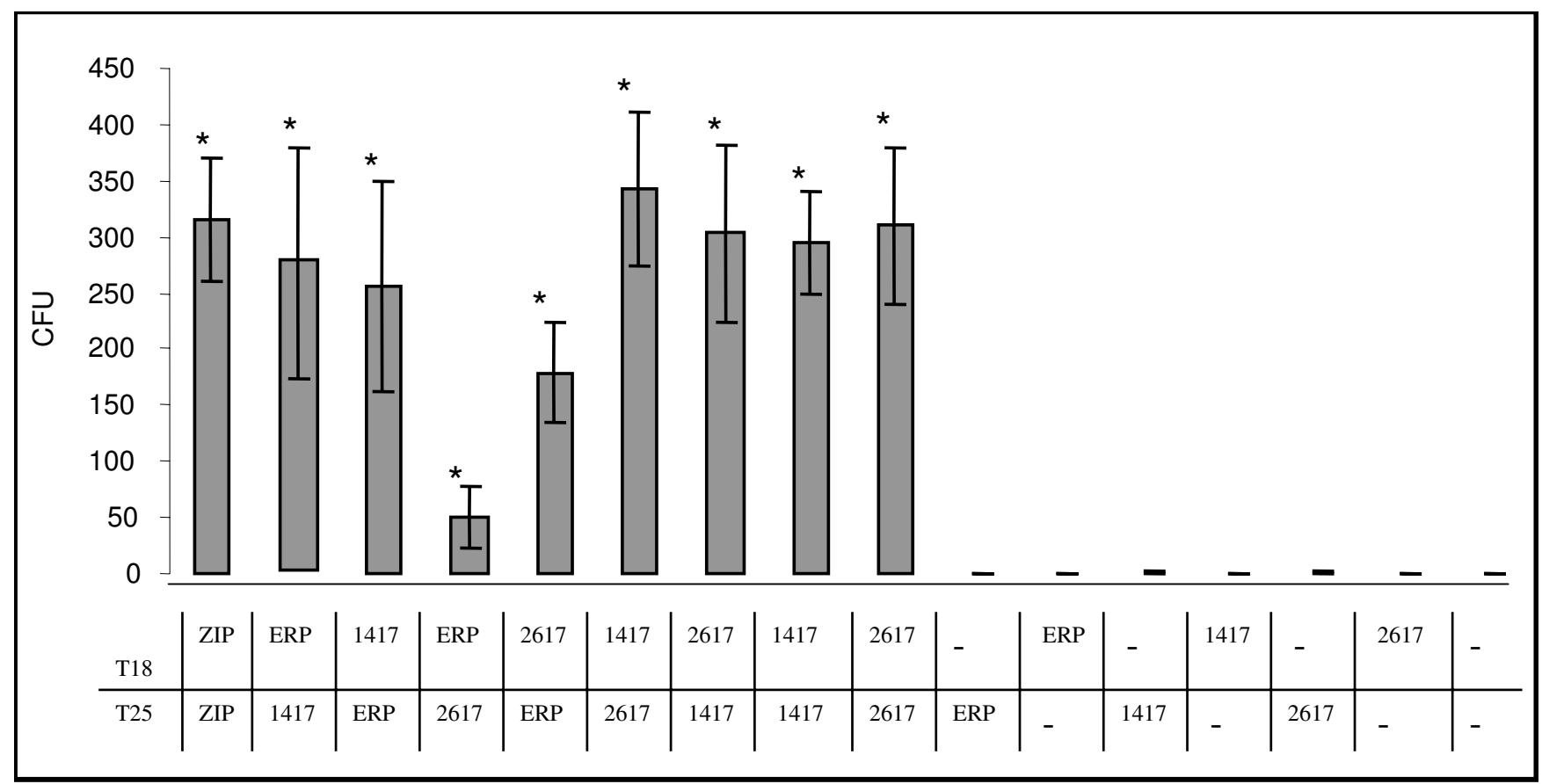

Figure I

In vivo interaction of Erp, RvI4I7 and Rv26I7c. E. coli BTH IOI cells were transformed with the plasmids described in the figure and plated in $\mathrm{M} 63$ medium supplemented with lactose. Colonies were counted after five days of culture. Triplicate plates were prepared. The bars represent the mean number of colonies \pm S.D. *Significantly different $(P<0.05)$ from the value of negative controls as calculated by the Student's t test. ZIP: positive control, I4 I7: Rv14I7, 26I7: Rv26I7c, ERP: Rv38I0.

\section{RvI4I7 and Rv26I7c interact with each other}

The binding of Erp to two different proteins raised the question whether these two proteins are able to interact with each other. In order to clarify this point, the binding between Rv1417 and Rv2617c was addressed by using the bacterial two-hybrid system. These experiments were facilitated by the availability of constructs with each gene in both vectors that had been prepared for the present work. Plasmids (encoding protein fusions of Rv1417 and Rv2617c with T25 and T18 polypeptides) were used to transform $E$. coli BTH101 cells. All plasmid combinations were subjected to a quantitative screening on selective medium plates. Each hybrid protein tested was able to associate with the other partners (Fig. 1 and Additional file 1). Indeed, both Rv1417 and Rv2617c exhibited strong self-associations, thus suggesting homodimer complex formation of these proteins (Fig. 1 and Additional file 3). The Erp fusions, however, were impaired in self-association (data not shown). None of the hybrid proteins gave a significant complementation signal when tested either with control T25 and T18 polypeptides or with unrelated proteins, like lipoprotein P27 [9].

\section{The carboxy-terminal domain of Erp is not relevant for protein interactions}

In an effort to map the Erp region involved in protein-protein interactions, we assessed the capacity of each Erp domain to bind both Rv1417 and Rv2617c. Firstly, we focused on determining whether the carboxy-terminal region of Erp, which is involved in the association with the cell wall [10], contained a binding domain. The full sequence of the erp gene was divided in two regions at the base number 528, and fusions of both regions to the T18 encoding sequence were generated. The resulting hybrid proteins were then tested in two-hybrid complementation assays with Rv1417 and Rv2617c fused to the T25 fragment. Deletion of the carboxy-terminal region of Erp did not affect its association with Rv1417 and Rv2617c, thus suggesting that this region of the protein is not essential for this interaction (Fig. 2).

As a second step, the interacting region of Erp was further divided at aa 80, and fusion proteins of the amino-terminal and central domains of Erp with T18 polypeptide were generated. When separated, the amino-terminal and the central domains were unable to interact with Rv1417 and Rv2617c (data not shown). Only the protein deleted in the carboxy-terminal region showed a level of interaction comparable to, or even higher than, that of the full-length protein.

\section{Interaction of Erp members from Mycobacterium smegmatis and Mycobacterium leprae}

As mentioned in the introduction section of this work, Erp carboxy- and amino-terminal domains are fully conserved, 


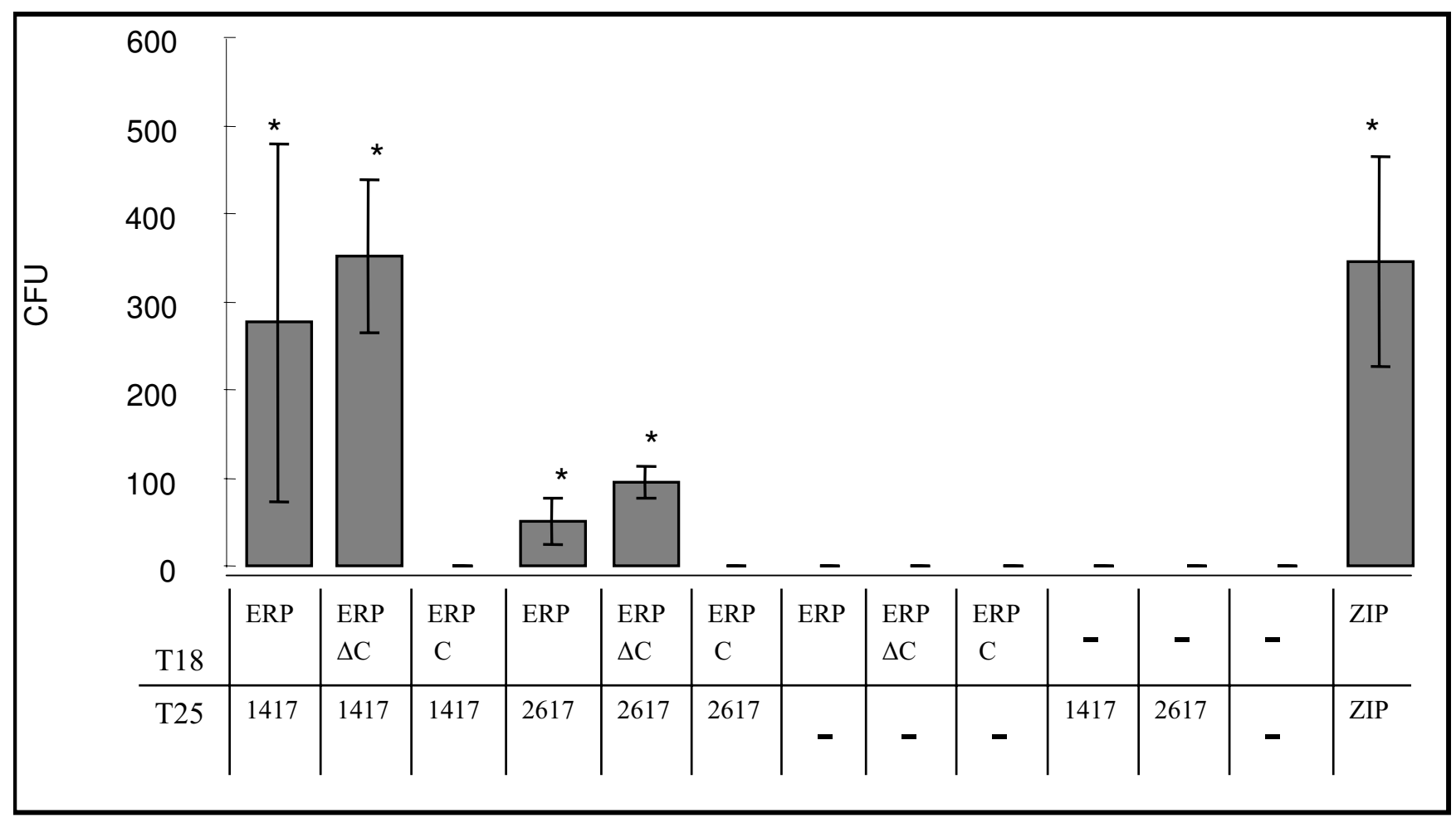

Figure 2

Mapping of the Erp interacting domain. The experiment was performed as in Fig. I. ZIP: positive control, I4I7: RvI4I7, 2617: Rv26I7c, ERP: Rv3810, ERP C: carboxi-terminal domain of Erp, ERP $\Delta$ C: Erp with its carboxy-terminal domain deleted.

while the central region shows polymoprhism among mycobacterial species, with respect to the number and quality of repeats. While M. leprae has four repeats, M. smegmatis has twenty-six, half of which contain two mismatches [4]. Therefore, it was plausible that the interaction with Rv1417 and Rv2617c was affected by the number of repeats. In order to evaluate this assumption, the interaction of M. smegmatis and M. leprae Erp homologues with both Rv1417 and Rv2617c was assayed. M. leprae Erp (Ml Erp) was unable to associate with either Rv1417 or Rv2617c. M. smegmatis Erp (Ms Erp) showed interaction with Rv1417 but completely failed to bind Rv2617c (Fig. 3). Although we can not exclude the possibility that the lack of interaction of Rv1417 and Rv2617c with the Erp member from M. leprae was due to a misfolding of the T25-Ml Erp protein, the impossibility of this fusion protein and of the T25-Ms Erp protein to interact with Rv2617c is interesting since it correlates with the absence of a functional $R v 2617 c$ gene in the $M$. leprae and $M$. smegmatis genomes (see below). On the other hand, the interaction of Rv1417 with the Erp member of M. smegmatis, but not with the one from $M$. leprae, indicates that the number and sequences of the PGLTS repeats are relevant for the occurrence of such interaction.

5. Erp, RvI4I7 and Rv26I7c are located in close proximity In order to determine the localization of the potential protein complex Erp-Rv1416-Rv2617c in mycobacterial cells, we first performed an in silico search for protein domains in Rv1417 and Rv2617c. Sequence analysis with InterProScan [11] recognized a signal-peptide domain in Rv1417 and Rv2617c. However, this was not confirmed by SOSUI or SignalP $[12,13]$, two different software programs that perform signal peptide predictions. Both SOSUI and TMHMM [14] predicted the presence of two and three transmembrane helices for Rv1417 and Rv2617c, respectively. The analysis performed with the SOSUI server showed two transmembrane helices (encompassing positions: 22-44 and 51-72) in Rv1417 and three transmembrane helices (encompassing positions: 19-41, 81-103 and 117-139) in Rv2617c. TMHMM predicted that the probability of an extracellular location for the C-terminal region of Rv1417 was 0.36 and 0.64 for a cytosolic orientation. The predicted probability for an outward orientation for the C-terminus of Rv2617c was approximately 0.82 . These results indicate a probable membrane localization of these proteins. To confirm these predictions, we performed immunolocalization of the proteins in subcellular compartments and in culture supernatants by using specific antibodies. Rv1417 and Rv2617c were expressed as a fusion to the myc epitope (see Materials and Methods) to allow their detection in mycobacterial cells. Since attempts to detect Rv1417-Myc in M. tuberculosis were unsuccessful, probably due to a very low expression of the recombinant protein, the fused protein was expressed in 


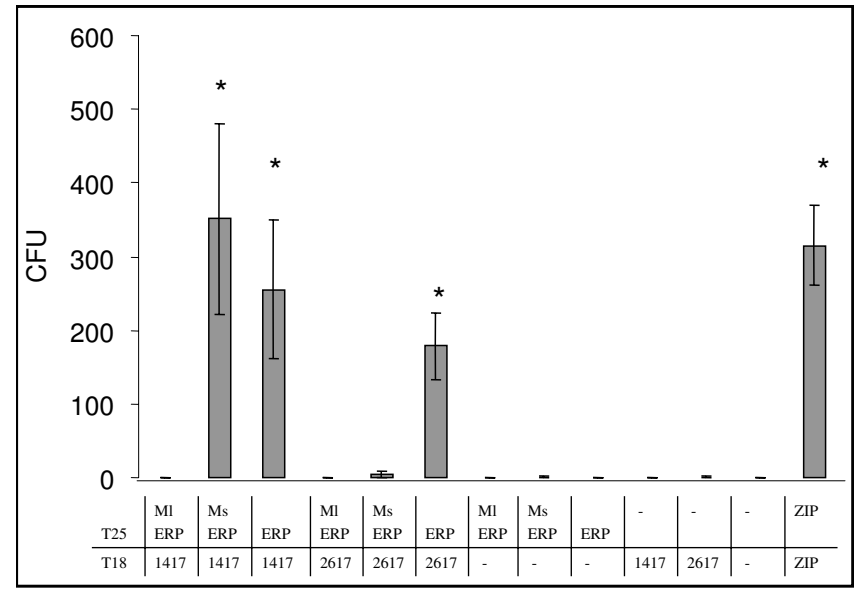

Figure 3

Interaction of Erp members from $M$. smegmatis and M. leprae with RvI4I7 and Rv26I7c. The experiment was performed as in Fig. I. ZIP: positive control, I4I7: RvI4I7, 2617: Rv26I7c, ERP: Rv38I0. MI ERP: M. leprae Erp homologue (ML009I), Ms ERP: M. smegmatis Erp homologue (MSMEG6405).

the M. smegmatis strain $m^{2} 155$. Figure 4 shows the localization of Rv1417-Myc and Rv2617c-Myc in the membrane fraction of recombinant $M$. smegmatis and $M$. tuberculosis, respectively, but not in those transformed with the empty vector. Therefore, the co-localization of Rv1417 and Rv2617c suggests that protein-protein associations may take place in the cell envelope. In agreement with previous studies $[1,2]$, Erp was identified in the cellular wall fraction and culture supernatant of M. tuberculosis by using a monoclonal specific antibody [5]. The absence of reacting bands in a P36-deficient $M$. bovis strain verified the antibody specificity.

\section{Characterization of interacting proteins}

In a BLAST [15] comparison of the predicted amino acid sequences of Erp interacting proteins, Rv1417 appeared to be conserved among the Mycobacterium genus and showed similarity to hypothetical membrane proteins from other bacterial species, such as Rhodococcus sp (identity 48\%, similarity: 67\%), Corynebacterium ammoniagenes (identity: 37\%, similarity: 58\%, with RibX protein), Corynebacterium diphterae (identity: 35\%, similarity: 59\%), Streptomyces coelicolor (identity: 34\%, similarity: 55\%) and Streptomyces ambofaciens (identity: 34\%, similarity: 57\%). Conversely, the $R v 2617 c$ gene was observed to be disseminated only among members of the M. tuberculosis complex (MTC). A pseudogene similar to $R v 2617 c$ was observed to be present in the $M$. leprae genome. The deduced amino acid sequence of $R v 2617 c$ showed similarity to hypothetical membrane proteins from Rhodococcus sp (identity: 48\%, similarity: 67\%), Nocardoides sp (identity: 61\%, similarity:

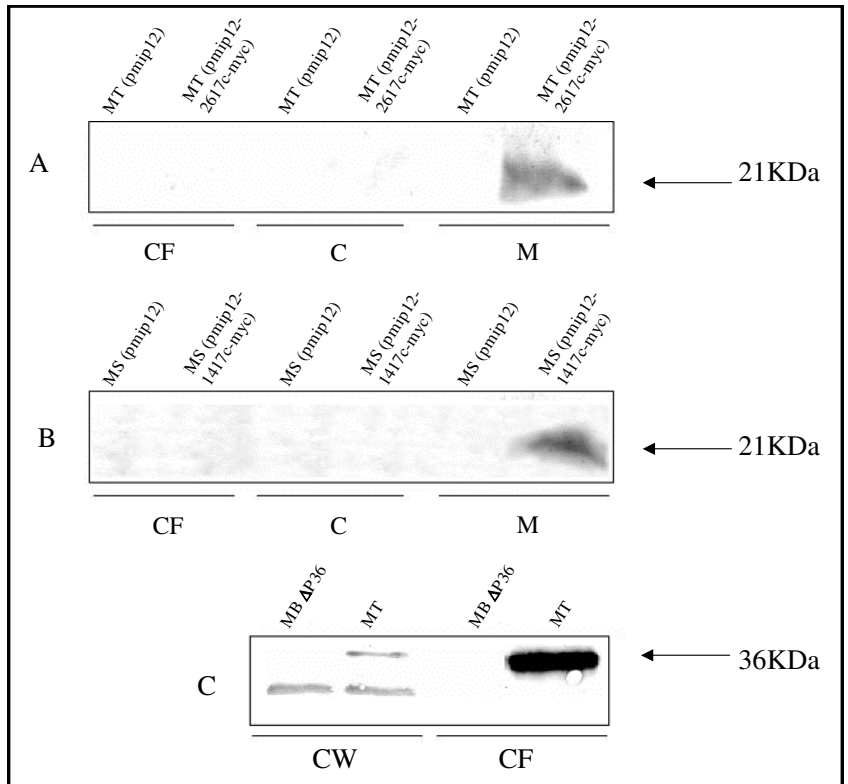

Figure 4

Cellular localization of Rv I4I7, Rv26 I 7c and Erp. Myc-RvI4I7 and Myc-Rv26I7c were expressed in M. smegmatis and $M$. tuberculosis, respectively. Proteins from culture filtrates (CF), cytoplasm $^{\odot}$, plasma membrane (M) and cell wall (CW) were extracted from the strains indicated above each lane and separated by I2\% SDS-PAGE. Bands were detected either with anti-Myc Mab (Sigma-Aldrich) at a I:I00 dilution (A and $\mathrm{B}$ ) or with anti-P36/Erp Mab [5] at I:500 dilution ${ }^{\odot}$. MT: M. tuberculosis, MT (pmip I2-26I7c-myc): M. tuberculosis (pmip |2-26I7c-myc), MT (pmip |2): M. tuberculosis (pmip I2), MS (pmip I2-14|7-myc): M. smegmatis (pmip I2-I4I7-myc), MS (pmip I2): M. smegmatis (pmip I2), MB P36: M. bovis $\triangle$ P36.

72\%), and Arthrobacter sp (identity: 56\%, similarity: $72 \%)$.

In order to experimentally analyse the distribution of $R v 2617 c$ and $R v 1417$ in the MTC, PCR assays using specific primers were performed on genomic DNA from MTC species. DNA fragments of expected size were obtained for each gene in all species studied (Fig. 5A). In addition, the transcription of Rv1417 and Rv2617c during the in vitro culture of $M$. tuberculosis was demonstrated by RT-PCR (Fig. 5B). These results suggest that Rv1417 and Rv2617C are functional genes conserved in the MTC.

We investigated the neighbourhoods of $R v 1417$ and $R v 2617 c$, as well as of their orthologues, with the aim of obtaining clues regarding the biological role of these genes. While examination of the genomic location of the $R v 2617 c$ and its orthologues did not reveal any particular feature, we found that Rv1417 and its orthologues are upstream flanked by genes encoding for proteins involved in riboflavin synthesis. Riboflavin operons with a similar 


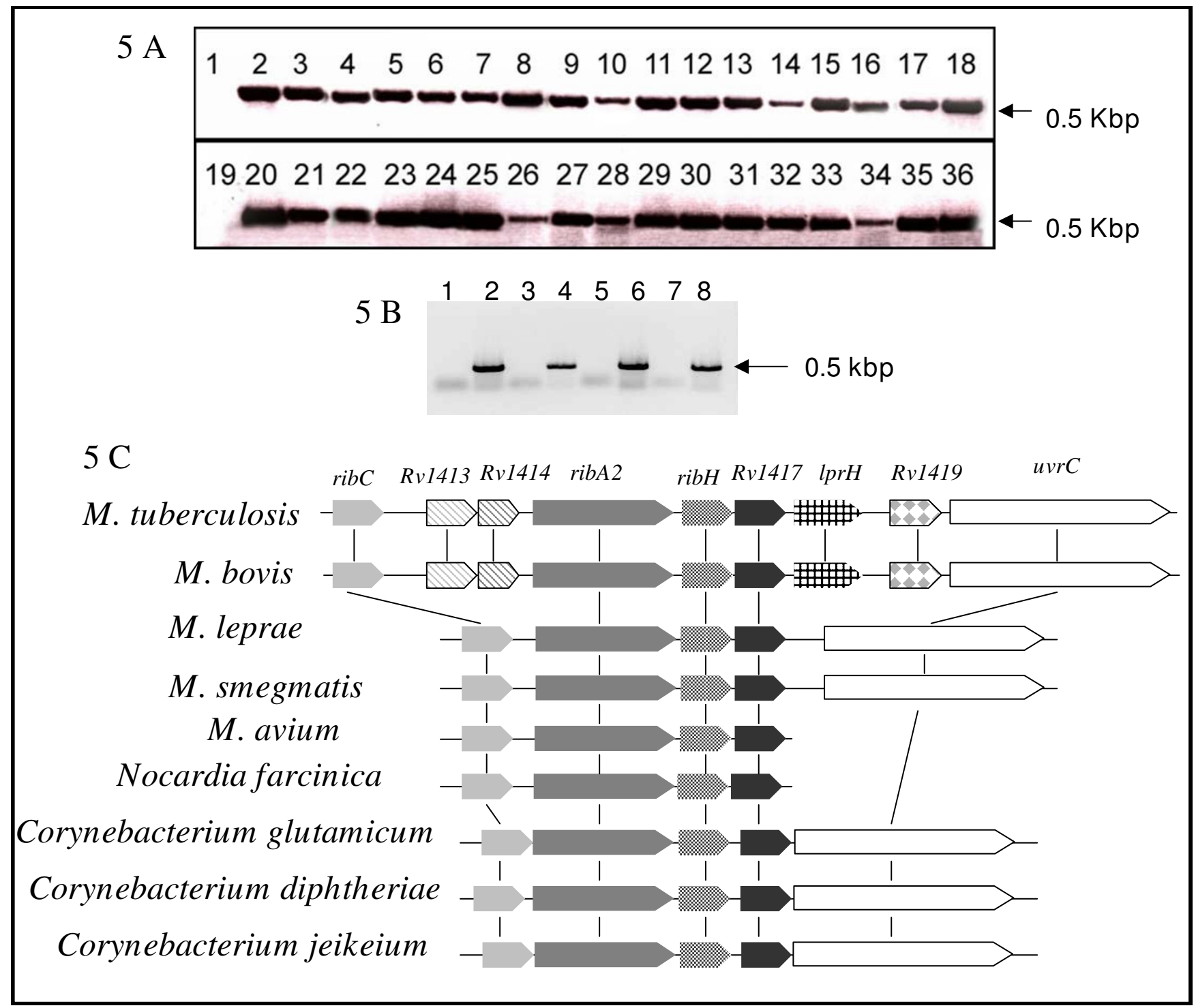

Figure 5

Genetic characterization and expression studies of $R v / 4$ I7 and Rv26I7c. A. Distribution of Rv/4I7 and Rv26I7c in species of the MTC. PCR amplifications of Rv/4/7 (lanes I-I8) and Rv26/7c (lanes 19-36) were performed with pairs of primers 14I7pktup/I4I7pktlow and 26I7pktup/26I7pktlow (table I), respectively, and using the following genomic DNA as template: lanes 2 and 20, M. tuberculosis H37Rv; lanes 3-7 and 2I-25, M. microti isolates; lanes 8-12 and 26-30, M. pinnipedii isolates; lanes I3-17 and 3I-35, M. bovis isolates; lanes I8 and 36, M. caprae isolate. PCR negative controls were included (lanes I and 19). Arrows indicate the size of the bands. B. Transcription of Rv/4I7 and Rv26I7c in M. tuberculosis. The transcription of Rv/4I 7 (lanes I-4) and Rv26I7c (lanes 5-8) was studied by RT-PCR assays using the pairs of primers I4I7pktup/ I4I7pktlow and 26I7pktup/26I7pktlow (table I), respectively. Lanes I and 5, PCR negative controls; lanes 2 and 6, M. tuberculosis DNA (positive control); lanes 3 and 7, RT-PCR amplifications without RT; lanes 4 and 8, RT-PCR amplifications with RT. Arrow indicates the size of the bands. C. Genomic organization of Rv I4I 7 homologous loci in Actinomycetales. Schematic representation of genes encoding conserved proteins in the neighbourhood of Rv / 4 I 7 in Actinomycetales genomes. Genes encoding homologous proteins are depicted in colours or patterns. Comparative genomic analysis was carried out with the STRING software and BLASTP analysis of the protein sequences deduced from genomic data bases.

structure, containing an Rv1417-like gene, were identified in all Mycobacterium species whose genomes were sequenced, as well as in other species of Actinomycetales genera (Fig. 5C). In addition, RibX, whose gene is a puta- tive member of the riboflavin operon of C. ammoniagenes, showed similarity to $\operatorname{Rv} 1417\left(E=e^{-22}\right)$. Although the role of RibX in riboflavin synthesis remains elusive, a DNA fragment that includes part of the ribX gene was demon- 
strated to be involved in riboflavin production [16]. To determine whether $R v 1417$ is co-transcribed with $R i b H$, a gene encoding a probable riboflavin synthase beta chain in M. tuberculosis and M. bovis strains, RT-PCR assays were performed by using primers that map across the two adjacent genes. The RT-PCR products shown in figure 6 indicate that $R v 1417$ and $R i b H$ are transcribed to a single mRNA molecule. Based on these results, we propose $R v 1417$ as part of the riboflavin operon in M. tuberculosis.

\section{Discussion}

The availability of the M. tuberculosis genome sequence has provided us with new information, knowledge and understanding of the biology of this major pathogen as well as raised a number of questions concerning the roles and functions of a large group of putative unknown proteins, in which Erp/P36 is included. Here we present findings that could contribute to decipher the function of Erp in M. tuberculosis. The construction of a two-hybrid library expressing M. tuberculosis proteins enabled us to identify two Erp interacting proteins, Rv1417 and Rv2617c. We found that Rv1417 and Rv2617c are similar to hypothetical membrane proteins from other bacterial species but not to characterized proteins.

By BLAST searches, we identified Rv1417 orthologues in several mycobacterial species, including M. smegmatis and M. leprae. The presence of Rv2617c seems to be restricted to the M. tuberculosis complex, as no functional orthologues were identified in non-tuberculous species while $R v 2617 c$ genes were found by PCR in Mycobacterium

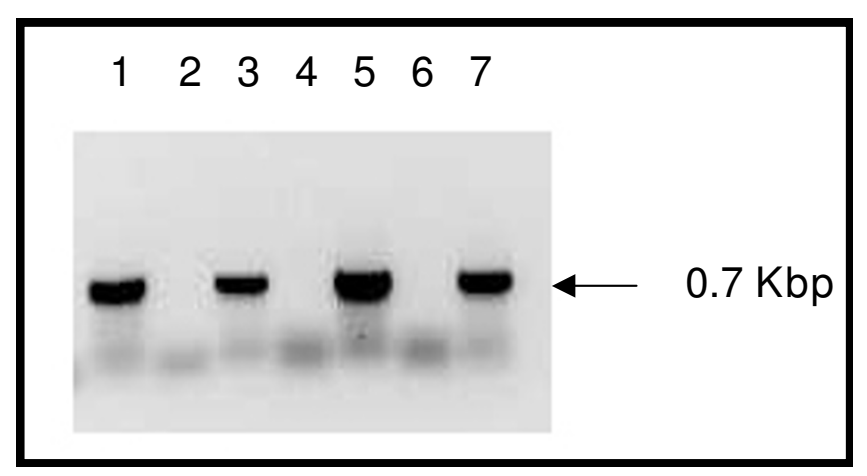

Figure 6

RT-PCR analysis of RvI4I7 and RibH in M. tuberculosis and $\boldsymbol{M}$. bovis. Co-transcription of $\mathrm{Rv} / 4 / 7$ and $\mathrm{RibH}$ was studied by RT-PCR assays. Total RNA from M. tuberculosis H37Rv (lanes I and 2) and from M. bovis AN5 (lanes 3 and 4) was reverse-transcribed and amplified with the primers LeftOPI4I7/RightOPI4I7 (table I). Lanes I and 3, RT-PCR amplification with RT; lanes 2 and 4, RT-PCR amplification without RT; lane 5, M. tuberculosis DNA (positive control); lane 6, PCR negative control; lane $7 \mathrm{M}$. bovis DNA (positive control). Arrow indicates the size of the bands. microti, Mycobacterium pinnipedii, Mycobacterium canetti and $M$. bovis genomes.

By protein-protein interactions we also demonstrated association between Rv1417 and Rv2617c. Importantly, in all cases, complementation between the T25 and the T18 hybrids was detected in both configurations, that is, when the given proteins were fused to either the T25 or the T18 polypeptides. Moreover, both Rv1417 and Rv2617c, but not Erp, showed self-interaction, indicating that the former are able to generate homodimers. Taken together, these results show that it is likely that the three proteins form a heteromultimeric protein complex.

However, at this point of the investigation we do not know whether more than two units of Rv1417 and Rv2617c are assembled in the putative multi-protein complex.

Since the signal sequence of Erp was present in the T25 and T18 fusions, we cannot rule out the possibility that interacting proteins are localized in the periplasm of $E$. coli. However, based on the fact that the bacterial twohybrid system allows the detection of protein interactions that occur either in the cytoplasm or at the inner-membrane level [7], this possibility is very unlikely.

Kocincova et al. have shown that the Erp protein is anchored to the surface of the bacterium by a carboxy-terminal hydrophobic domain and that it is easily released into the supernatant fraction [10]. From these data these authors proposed that Erp uses the carboxy-terminal domain to interact with some other molecules of the cell wall to achieve its correct structure. This finding led us to think that Rv1417 and Rv2617c might have the potential to anchor Erp by interaction with its carboxy-terminal domain. Nevertheless, here we demonstrated that this domain does not contribute to the interactions, thus suggesting that the membrane proteins Rv1417 and Rv2617c are not involved in the attachment of Erp to the cell surface. Remarkably, it has been demonstrated that the carboxy-terminal region of Erp is not essential for restoring the virulence and tissue damage of an erp-mutant strain of M. tuberculosis [17]. Therefore, based on the facts that this carboxy-terminal region, which is conserved through Mycobacterium species, is not implicated in protein interaction or virulence, one may speculate that the virulence properties of Erp are related with its capability of interaction. We found that the region involved in the interaction with Rv1417 and Rv2617c was located in the amino-terminal and central domains of Erp. However, we do not know whether the interactive domains are mapped at the central repetitive region and the amino-terminal region is relevant for protein folding, contributing to the protein 
association, or whether additional binding domains are present at the amino-terminal region.

The interaction of Rv1417 and Rv2617c with the repeat central domain of Erp is intriguing because this domain appears to be associated with a role in virulence and tissue damage of M. tuberculosis in mice. Recently, de Mendoça Lima et al. [18] have shown that at early time-points of infection in lungs, Erp from M. smegmatis is not able to restore the wild type virulence of the erp-deficient $M$. tuberculosis strain, whereas Erp from pathogenic M. leprae induces a hypervirulent phenotype. In this work, we demonstrated that Erp from M. leprae (ML0091) was not able to interact either with Rv1417 or with Rv2617c, and that Erp from M. smegmatis (MSMEG6405) was able to interact with Rv1417 but not with Rv2617c. The absence of Rv2617c in species that do not belong to the MTC and the inability of both ML0091 and MSMEG6405 to interact with Rv2617c indicate that the Rv2617c-Erp interaction is restricted to species from the MTC. These interactions may have been acquired during divergent evolution when tuberculous Mycobacterium species arose. Thus, this work provides evidence suggesting multiple roles for members of the Erp family, which could explain why, being relevant for intracellular living, this proteins are present in saprophytic Mycobacterium species. On the other hand, the interaction of Rv1417 with the Erp member from M. smegmatis but not with the one from $M$. leprae suggests that the virulence role played by Erp orthologues is not related with their capacities of interaction with Rv1417. The investigation of such interactions in a variety of mycobacterial species will help to clarify this point.

Although the pairwise interactions of Rv1417, Rv2617c and Erp were clearly demonstrated, they may reflect a transient contact in an assembly pathway or stable interactions in a completed structure. In order to define this point, immunolocalization of the three proteins in the bacterial cell was investigated. In agreement with previous findings [1-3], Erp was localized mostly in culture supernatants, but also associated to the cell wall, whereas Rv1417 and Rv2617c were restricted to the cell membrane. This last finding is in agreement with the prediction of transmembrane helices in both Rv1417 and Rv2617c as determined by SOSUI and TMHMM software. An initial scan with InterProScan predicted a signal-peptide domain in these proteins that could not be confirmed neither by the specialized SOSUI nor by the SignalP programs. This is not unexpected since mycobacterial exported proteins without predicted signal sequences have been previously described [19]. Therefore, we postulate that Rv1417 and Rv2617c form a multimeric structure on the membrane which is, in turn, transiently associated to the Erp protein before being translocated to the cell envelope and the extracellular compartment. Although the experiments carried out in this study did not allow us to define whether Rv1417 and Rv2617c are localized in the cytoplasmic membrane or in the outer membrane, the former localization appears more plausible since these proteins are not predicted to be present in the outer membrane [20].

Our work shows that both Rv1417 and Rv2617c genes are transcribed in in vitro cultured $M$. tuberculosis and that $R v 1417$ is co-transcribed with the RibH gene, which is part of a riboflavin operon. We also found that $R v 1417$ orthologues are located in genomic regions that encode proteins that participate in the riboflavin metabolism in several species of Actinomycetales. Riboflavin (vitamin B2) is the precursor of the coenzymes flavin mononucleotide phosphate and flavin adenine dinucleotide phosphate, essential compounds for basic metabolism. It has been demonstrated that riboflavin biosynthesis is essential for in vivo survival of a number of bacterial species because of the scarcity of riboflavin in mammalian cells $[21,22]$. Although these findings strongly suggest a role of Rv1417 in riboflavin synthesis, more research is necessary to understand the association of riboflavin metabolism with the function of Erp and Rv2617c in M. tuberculosis, as well as to elucidate the biological significance of the protein interactions discovered in this study.

\section{Conclusion}

We identified interactive partners of Erp, an M. tuberculosis protein involved in virulence, which will be the focus of future investigation to decipher the function of the Erp family protein.

\section{Methods \\ Bacterial strains and culture media}

All cloning steps were performed in E. coli DH5 $\alpha$, and E. coli BL21(DE3) was used for recombinant protein production. Complementation assays were carried out with the E. coli BTH101 strain (F- cya-99, araD139, galE15, galK16, $r p s \mathrm{~L} 1$ (Strr $), h s d \mathrm{R} 2, m c r \mathrm{~A} 1, m c r \mathrm{~B} 1)$. E. coli strains were grown either in Luria-Bertani (LB) broth or on LB agar. Screening for the ability to ferment sugars was performed on M63 plates supplemented with $0.3 \%$ lactose. When necessary, ampicillin at $100 \mu \mathrm{g} / \mathrm{ml}$ and kanamycin at 50 $\mu \mathrm{g} / \mathrm{ml}$ were added to the media. Mycobacterium strains were grown in either Middlebrook 7H9 medium supplemented with $0.05 \%$ Tween 80 or Middlebrook $7 \mathrm{H} 11$ medium, both supplemented with ADC (albumin -1 $0.5 \%$, dextrose $0.4 \%$ ), and $0.5 \%$ glycerol. When necessary, $20 \mu \mathrm{g}$ kanamycin $\mathrm{ml}^{-1}$ was added to the media. Electrocompetent $M$. tuberculosis and $M$. smegmatis cells were prepared and transformed by electroporation, as described by Parish and Stoker [23]. 


\section{Construction of an M. tuberculosis library in a pUTI8c plasmid}

M. tuberculosis chromosomal DNA was partially digested with AluI. Then, 0.5- to 1-kbp purified DNA fragments were ligated to pUT18c plasmids digested with Sma I. The ligation mixture was electroporated into E. coli DH5 $\alpha$ strain. Transformants were suspended in LB medium containing ampicillin, and plasmid DNA from this library was prepared. Analysis of randomly selected plasmids from individual clones showed that the average size of inserts was 0.5-1 kbp (data not shown). Plasmids were purified from pooled clones to generate a plasmid library.

\section{Construction of bait and prey plasmids}

The entire open reading frames (ORFs) of Erp, P27, Rv1417 and Rv2617c (of M. tuberculosis) as well as the ORF of ML0091 (of M. leprae) and MSMEG6405 (of M. smegmatis) were PCR-amplified from their corresponding genomes and cloned as fusion to the T25 subunit of the adenylate cyclase of Bordetella pertussis into the bait vector pKT25 [7]. DNA fragments encoding for the amino-terminal region, the central domain, the carboxy-terminal region, the full-length Erp protein, and a version of erp deleted in the sequence encoding the carboxy-terminal region were PCR-amplified from $M$. tuberculosis chromosome and amplicons were cloned as fusions to the T18 subunit of the adenylate cyclase of $B$. pertussis into the prey vector pUT18C [7]. Details of primers used and plasmids generated are depicted in table 1.

\section{Identification of gene products interacting with Erp by a bacterial two-hybrid assay}

About $1 \mu \mathrm{g}$ of plasmid DNA library was used to co-transform E. coli BTH101 competent cells harbouring the T25Erp bait plasmid. Co-transformants were selected on M63 plates containing $0.3 \%$ lactose, ampicillin, kanamycin and $40 \mu \mathrm{g} / \mathrm{ml}$ of X-gal (5-bromo-4-chloro-3-indolyl-b-Dgalactopyranoside). A small aliquot of the transformants was plated on rich LB medium containing ampicillin and kanamycin to select the presence of pUT18C derivatives. The total number of transformants deduced from these experiments demonstrated high efficiency of transformation of E. coli cells (data not shown). Blue colonies on M63 medium appearing after 5 days of incubation were assumed to contain pUT18C derivatives coding for potential proteins that interact with Erp. The pUT18C derivatives were isolated from these clones and reintroduced either into BTH101 cells containing T25-Erp or into BTH101 cells containing the empty pKT25 vector (as a negative control) to confirm the interactions and to exclude false positives. Hybrid plasmids from positive clones were sequenced, and genes coding for putative interactors were identified by BLAST searches in the $M$. tuberculosis genome database [8].

\section{Protein-protein interaction assays}

E. coli BTH101 competent cells (which had a level of competency of $1 \times 10^{7}$ ) were co- 6 transformed with bait and prey plasmids ( $1 \mu \mathrm{g}$ of each plasmid) and $1 \times 10^{6}$ cells were plated on M63 plates containing lactose, ampicillin, kanamycin and X-gal. The strength of interaction was assayed by counting the number of colonies on M63 plates.

In addition, controls of the efficiency of transformation were performed by plating $1 \times 10^{6}$ cells from each transformation mix on LB medium containing ampicillin and kanamycin. The number of colonies grown in the rich medium was approximately the same for all the reactions.

The interaction of fusion proteins encoded in T18-Zip and T25-Zip plasmids was used as a positive control.

\section{Statistical analysis}

Means were tested for differences with Student's t test. Values were determined to be statistically significant at $\mathrm{P}<$ 0.05 .

\section{Computer analyses}

All identified ORFs were subjected to bioinformatic analysis including similarity searches, protein domain determination and genomic structure. Sequence similarity searches were performed by BLASTP [15]. The InterProScan software was used to search for conserved domains in the proteins against the InterPro database [11]. Transmembrane helix predictions were performed using the TMHMM Server [14]. Comparative genomic analysis was carried out with the String software [24] and BLAST analysis of the genome sequences.

\section{RT-PCR}

RT-PCR reactions were performed from DNA-free RNA (1 $\mu \mathrm{g}$ ) extracted from middle logarithmic-phase cultures of M. tuberculosis H37Rv as described previously [25]. The primers used in each assay are summarized in Table 1.

\section{Protein localization}

Since attempts to raise antibodies against Rv1417 and Rv2617c were unsuccessful, the myc tag sequence was fused to the 3' end of both Rv1417 and Rv2617c and an anti-myc monoclonal antibody was used to recognise the recombinant protein in the recombinant mycobacterial strains. The full-length sequences of $R v 1417$ and $R v 2617 c$ were cloned into the ImpactVector 1.1-tag plasmid (Wageninger UR). The myc-tagged genes were PCR-amplified from the resulting plasmids and cloned in pmip12 [26] (see Table 1). Recombinant plasmids were used to transform M. tuberculosis $\mathrm{H} 37 \mathrm{Rv}$ and M. smegmatis competent cells as described above. Subcellular fractioning of $\mathrm{Myco}$ 
Table I: Plasmids and primers used in this study

\begin{tabular}{|c|c|c|c|}
\hline Plasmid ** & Primers & Sequence $5^{\prime}-3^{\prime a}$ & Origin \\
\hline \multirow[t]{2}{*}{ T25-Erp } & Erpbaitup & ctgcaggggtgccgaaccgacgccga & This study \\
\hline & ERPbaitlow & ggtaccttaggcgaccggcacggt & \\
\hline \multirow[t]{2}{*}{$T 25-1417$} & 14|7pktup* & ggatcccgtgaccgccgcaccgaac & This study \\
\hline & 1417pktlow* & ggtacctcagcgggegcacaggtc & \\
\hline \multirow[t]{2}{*}{ T25-2617 } & 2617 pktup* & ctgcagggatgagcatcagaccaacg & This study \\
\hline & 2617 pktlow* & ggtaccttaaggccgcccgatgcc & \\
\hline \multirow[t]{2}{*}{ T25-MIErp } & ERPlepraeup & ggatccagtgccgaaccgacgccgatg & This study \\
\hline & ERPlepraelow & ggatccctacgtgacaggaatcagtg & \\
\hline \multirow[t]{2}{*}{ T25-MsErp } & ERPsmgupok & ggtacctgtgccgaaccgccgtcga & This study \\
\hline & ERPsmglow & ggtacctcagggtcgccgcgat & \\
\hline \multirow[t]{2}{*}{ TI8- ErpA } & ERPputup & ctgcaggggtgccgaaccgacgccga & This study \\
\hline & ERPaminochicolow & ggtaccctaggtcaggctgggcaccgg & \\
\hline \multirow[t]{2}{*}{ TI8-PGLTS } & ERPPGLTSUP & ctgcagggggagcggcgatgccagcac & This study \\
\hline & ERPPGLTSIow & ggtaccctattcgttggcgccgcccag & \\
\hline \multirow[t]{2}{*}{ TI8-ErpC } & ERPCup & ctgcaggatcccgattacgacgccggt & This study \\
\hline & ERPputlow & ggtaccttaggcgaccggcacggt & \\
\hline \multirow[t]{2}{*}{ TI8-Erp } & ERPputup & ctgcaggggtgccgaaccgacgccga & This study \\
\hline & ERPputlow & ggtaccttaggcgaccggcacggt & \\
\hline \multirow[t]{2}{*}{$\mathrm{TI}$ I8-Erp $\Delta \mathrm{C}$} & ERPputup & ctgcaggggtgccgaaccgacgccga & This study \\
\hline & ERP A low & ggtaccctattcgttggcgccgcccag & \\
\hline \multirow[t]{2}{*}{ pmip | 2-14|7-myc } & up 1417 myc & ggtaccgtgaccgccgcaccga & This study \\
\hline & LowMyc & ggtaccttagtgatggtgatggtg & \\
\hline \multirow[t]{2}{*}{ pmip | 2-26I7c-myc } & up $2617 \mathrm{cmyc}$ & ggtaccatgagcatcagaccaac & This study \\
\hline & LowMyc & ggtaccttagtgatggtgatggtg & \\
\hline pmip I 2 & - & - & {$[26]$} \\
\hline \multirow[t]{3}{*}{ ImpactVector I.I } & - & - & Wageninger UR \\
\hline & LeftOP I $417^{* * * *}$ & cagctggcacggaaagat & This study \\
\hline & RightOP I 4 I $7 * * *$ & acgacacaccgatcacttca & \\
\hline
\end{tabular}

** Plasmids contain the sequence obtained by amplification with the pair of primers on the right column.

* Primers used in PCR or RT-PCR analysis.

*** Primers used only in RT-PCR analysis.

aRestriction enzyme sites are underlined.

bacterium strains was performed as described previously [9].

\section{Western blots}

Western blot assays were performed as described previously [27] with the following antibodies: anti-P36/Erp Mab (1:500) [5] and anti-myc monoclonal antibody (1:100/Sigma-Aldrich). Alkaline phosphatase-conjugated anti-mouse immunoglobulin G (1:2000/Sigma-Aldrich) was used to detect anti-myc and anti-P36/Erp antibodies.

\section{Authors' contributions}

LIK carried out the molecular biology and protein studies and participated in the draft of the manuscript. FCB and MVB carried out the RT-PCR assays and pull-down experiments, respectively. MS performed the informatics analy- 
sis. MPS helped in the elaboration of the manuscript. AAC and FB conceived the study and participated in its design and coordination and drafted the manuscript. All authors read and approved the final manuscript.

\section{Additional material}

\section{Additional file 1}

In vivo interaction of Erp, $R v 1417$ and $R v 2617 c$. The data provided shows the in vivo interaction between Erp-Rv1417, Erp-Rv2617c and Rv1417- Rv2617c using the bacterial two- hybrid assay.

Click here for file

[http://www.biomedcentral.com/content/supplementary/14712199-10-3-S1.doc]

\section{Additional file 2}

In vitro interaction of Erp with either $R v 1417$ or $R v 2617 c$ by pull down assay. The data provided shows the in vitro interaction between Erp-Rv1417 and Erp-Rv2617c using the GST-pull down assay.

Click here for file

[http://www.biomedcentral.com/content/supplementary/14712199-10-3-S2.doc]

\section{Additional file 3}

In vivo interaction of Rv1417 and Rv2617c. The data provided shows the ability of Rv1417 and Rv2617c to form homodimers using the bacterial two hybrid assay.

Click here for file

[http://www.biomedcentral.com/content/supplementary/14712199-10-3-S3.doc]

\section{Acknowledgements}

The present study was supported by SECyT grant Bid I728- PAV I 37 I/2, INTA grant AEBIO3454, and by CONICET PIP 578I. MPS, AC and FB are CONICET researchers. $L K$ is recipient of a fellowship from CONICET.

We thank Valeria Rocha for performing the plasmid constructions, and Luis Fernandez for the bibliography provided. We also thank Dr. Brennan and Dr. Spencer for providing us with M. leprae strain NHDP-63 genomic DNA (NHI Contract NOI-AI-25469), and Dr. Ladant for supplying the plasmids and strains required for the two-hybrid assay.

\section{References}

I. Berthet FX, Rauzier J, Lim EM, Philipp W, Gicquel B, Portnoï D: Characterization of the Mycobacterium tuberculosis erp gene encoding a potential cell surface protein with repetitive structures. Microbiology 1995, I 41:21 23-2130.

2. Berthet FX, Lagranderie M, Gounon P, Laurent-Winter C, Ensergueix $D$, Chavarot P, Thouron F, Maranghi E, Pelicic V, Portnoi D, Marchal $G$, Gicquel B: Attenuation of virulence by disruption of the Mycobacterium tuberculosis erp gene. Science 1998, 282:759-762.

3. Bigi F, Alito A, Fisanotti JC, Romano MI, Cataldi A: Characterization of a novel Mycobacterium bovis secreted antigen containing PGLTS repeats. Infect Immun 1995, 63:258I-2586.

4. de Mendonca-Lima L, Picardeau M, Raynaud C, Rauzier J, de la Salmoniere YO, Barker L, Bigi F, Cataldi A, Gicquel B, Reyrat JM: Erp, an extracellular protein family specific to mycobacteria. Microbiology 200I, I 47:23I5-2320.

5. Bigi F, Gioffre A, Klepp L, Santangelo MP, Velicovsky CA, Giambartolomei GH, Fossati CA, Romano MI, Mendum T, McFadden JJ, Cataldi A: Mutation in the P36 gene of textitMycobacterium bovis provokes attenuation of the bacillus in a mouse model. Tuberculosis 2005, 85:221-226.

6. Cosma CL, Klein K, Kim R, Beery D, Ramakrishnan J: Mycobacterium marinum Erp is a virulence determinant required for cell wall integrity and intracellular survival. Infect Immun 2006, 74:3|25-3|33.

7. Karimova G, Pidoux J, Ullmann A, Ladant D: A bacterial twohybrid system based on a reconstituted signal transduction pathway. Proc Natl Acad Sci 1998, 95:5752-5756.

8. M. tuberculosis genome database [http://genolist.pasteur.fr/ TubercuList/]

9. Bigi F, Espitia C, Alito A, Zumarraga M, Romano MI, Cravero S, Cataldi A: A novel 27 kDa lipoprotein antigen from Mycobacterium bovis. Microbiology 1997, I 43:3599-3605.

10. Kocincova D, Sonden B, de Mendonca-Lima L, Gicquel B, Reyrat JM: The Erp protein is anchored at the surface by a carboxy terminal hydrophobic domain and is important for cell-wall structure in Mycobacterium smegmatis. FEMS Microbiol Lett 2004, 23 I: $191-196$.

II. InterPro [http://www.ebi.ac.uk/interpro/]

12. Classification and Secondary Structure Prediction of Membrane Proteins [http://bp.nuap.nagoya-u.ac.jp/sosui/]

13. SignalP 3.0 Server [http://www.cbs.dtu.dk/services/SignalP]

14. TMHMM Server version 2.0 [http://www.cbs.dtu.dk/services/ TMHMM/]

15. Basic Local Alignment Search Tool [http:// www.ncbi.nlm.nih.gov/BLAST]

16. Koizumi S, Yonetani Y, Teshiba S: Process for producing riboflavin. U S Patent 1996.

17. Kocincova D, Sonden B, Bordat Y, Pivert E, de Mendonca-Lima L, Gicquel B, Reyrat JM: The hydrophobic domain of the Mycobacterial Erp protein is not essential for the virulence of Mycobacterium tuberculosis. Infect Immun 2004, 72:2379-2382.

18. de Mendoça-Lima L, Bordat Y, Pivert E, Recchi C, Neyrolles O, Maitournam A, Gicquel B, Reyrat JM: The allele encoding the mycobacterial Erp protein affects lung disease in mice. Cell Microbiol 2003, 5:65-73.

19. Di Giuseppe Champion PA, Cox JS: Protein secretion systems in Mycobacteria. Cell Microbiol 2007, 6: I376-1384.

20. Song $H$, Sandie $R$, Wang $Y$, Andrade-Navarro MA, Niederweis $M$ : Identification of outer membrane proteins of Mycobacterium tuberculosis. Tuberculosis 2008, 6:526-44.

2I. Fuller TE, Thacker BJ, Mulks MH: A riboflavin auxotroph of Actinobacillus pleuropneumoniae is attenuated in swine. Infect Immun 1996, 64:4659-4664.

22. Ashour J, Hondalus MK: Phenotypic mutants of the intracellular actinomycete Rhodococcus equi created by in vivo Himarl transposon mutagenesis. J Bacteriol 2003, 185:2644-2652.

23. Parish T, Stoker NG: Mycobacterial Protocols. In Method in Molecular Biology Volume 101. Edited by: Walker JM. New Jersey: Humana Press Totowa; 1998:129-144.

24. STRING - Proteins and their Interactions [http:// string.embl.de/]

25. Santangelo MP, Blanco FC, Bianco MV, Klepp LI, Zabal O, Cataldi AA, Bigi F: Study of the role of Mce3R on the transcription of mce genes of Mycobacterium tuberculosis. BMC Microbiol 2008, 8:38-46.

26. Le Dantec C, Winter N, Gicquel B, Vincent V, Picardeau M: Genomic sequence and transcriptional analysis of a 23-kilobase mycobacterial linear plasmid: evidence for horizontal transfer and identification of plasmid maintenance systems. | Bacteriol 200I, |83:2157-2|64.

27. Cataldi A, Romano Ml, Bigi F: A Western blot characterization of Mycobacterium bovis antigen recognized by cattle sera. Research in Microbiology 1994, 145:689-698. 